\title{
Introduction: why and how we mimic emotions
}

\author{
Agneta H. Fischer and Ursula Hess
}

Mimicry and its presumed neurological underpinnings in the form of mirror neurons have become a trending topic in the social, behavioural, and neurosciences during the past decades (e.g. Hess \& Fischer, 2013). The notion that minds can be shared by subtly imitating others is a challenging idea that sparks our imagination. For ages, philosophers and scientists have been intrigued by the question of how we come to understand what others think. How can we read the minds of others, how can we know what they feel and predict how they will react, and how can we relate to their pain and suffering or join in their victories and pride? Understanding of and empathizing with others appear to form the basis for human bonding and positive social interaction. Whereas shared goals or interests may lead to temporary coalitions and cooperation, understanding others' feelings and perspectives provides the basis for longterm and enduring social bonds.

One crucial aspect of the sharing of minds is the communication of emotions. We cannot be involved in relationships without emotions. We feel and express emotions towards the people we care about, and if people do not elicit any emotions in us, this implies that we feel indifferent towards them. Thus we get angry, irritated, worried, sad, or proud with the people to whom we feel connected; the stronger our concerns, the stronger the emotions, whether negative or positive. Not only do people express their emotions, they also tend to share them with their friends, family and colleagues. The communication of emotions is thus part of many daily interactions as well. A cashier smiles when handing back change, nurses express sympathy to patients, and managers show enthusiasm to motivate employees. The study of emotional expressions has a long tradition in psychology, starting with Darwin and then the seminal work by Paul Ekman; yet, for many years the question mainly focused on observers' ability to recognize the facial signals of emotion sent by an expresser. In this line of research the perceiver remained passive. 
Hatfield, Cacioppo, and Rapson (1994) were among the first to put the relationship between the perceiver and the perceived on the scientific agenda with the publication of their book Emotional Contagion. Emotional contagion is the sharing of emotional minds, and refers to the idea that we easily catch others' emotions. To illustrate the pervasiveness of this phenomenon, we only need to think about emotional crowds in panic, fright, or anger, or the pain we ourselves experience when watching the suffering of war victims and refugees on television. In their book Hatfield and colleagues identified several mechanisms that may lead to emotional contagion, of which mimicry has to date received most attention. Mimicry has been defined as the tendency to automatically imitate and synchronize facial expressions, vocalizations, postures, and movements with those of another person (see Hatfield et al., 1992). In other words, perceivers actively react to the emotional expressions of others and respond by showing matching - or sometimes contrasting - expressions. The tendency to mimic the expressions perceived in others can already be observed in small children and is assumed to have the important social functions of signalling affiliative intent and fostering rapport. For this reason, mimicry has been considered one of the cornerstones of successful and warm interactions.

Since then, an abundance of empirical research has been published on mimicry, investigating the determinants, boundaries, and effects of this phenomenon. We roughly distinguish between behavioural and emotional mimicry, the latter focusing on the mimicry of others' non-verbal behaviours that signal emotional meaning, such as a smile. The topic of the present volume is emotional mimicry. Several experts in this field reviewed the underlying processes and contextual boundaries of mimicry in order to better understand its functions in realizing the sharing of emotional perspectives. To date there is no clear picture regarding the conditions under which emotional mimicry is shown, or the situations in which it may be inappropriate. This is partly due to the fact that the literature on emotional mimicry is distributed over various subdisciplines in psychology and neuroscience. Thus, relevant studies have been published in diverse outlets across the domains of social psychology, emotions, neuroscience, biological psychology and psychophysiology, and even psycho-endocrinology. As a consequence the literature on this topic tends to be theoretically disjointed.

The aim of the present volume is to review and evaluate the state of the art in this research domain and to set out future directions. The only previous book addressing these issues, Emotional Contagion by Hatfield et al. (1994), was published more than 20 years ago, and since then many studies have been published and theoretical perspectives have been proposed. The present volume aims to reflect the state of the art on 
different aspects of mimicry, ranging from different factors and contexts affecting mimicry, different forms of mimicry (e.g. cross-channel mimicry), to the different functions of mimicry. The common thread in this volume is that emotional mimicry is a complex phenomenon influenced by context, usually a social exchange, which is crucial for when mimicry occurs and how it affects people.

\section{Overview}

\section{Mimicry, empathy, and the sharing of mind}

Mimicry is closely related to empathy, but there have been different ways in which this relation has been conceptualized. Mimicry can be seen as one of the causal paths to empathy (e.g. Hatfield et al., 1992), or as an early stage of empathy. In Chapter 1 Oatley refers to Donald (1991), who describes the mimetic stage as a crucial stage in the development of the mind, and as a preadaptation for language. This is also apparent in young children who mimic adults' facial expressions, signalling that they share the other's mind, before they are able to talk. Mimicry can thus be seen as an early stage in the development of empathy, preceding shared attention, shared intentions, and perspective taking. These are different paths all leading to the sharing of minds. Empathy is the sharing of emotions, which forms the building blocks of social life. Sharing of emotions is important for social coordination of plans in everyday life, but also for the appreciation of art, more specifically literature. Through art we identify with the protagonist and share life stories in films or novels.

Schuler, Mohnke, and Walter (Chapter 9) further differentiate between different forms of empathy, namely affective and cognitive empathy. Both forms of empathy differ from mimicry, although the neurological underpinnings are very similar. The core empathy regions comprise the AI (Anterior Insula) and ACC/MCC (Anterior and Medial Cingulate Cortex, $\mathrm{XE}$ " $\mathrm{ACC} / \mathrm{MCC}^{\prime}$ ), which integrate information across various domains and allow selection of prospective responses. Two brain circuits, involving the mirror neuron system and the mentalizing system, have been associated with two routes to empathy, namely top-down and bottom-up. The first route involves more cognitive perspective taking, and the second route involves the mirror neuron system, eliciting automatic (mimicry) responses to affective stimuli. Although these routes can be distinguished, it should be clear that they are not independent of each other.

Not only do empathy and mimicry seem to have similar neurological underpinnings but individual differences in empathy can also be seen as determinant of individual differences in mimicry. In Chapter 7, SonnbyBorgström reports evidence that individual differences in empathic 
ability, as measured with self-reports, are related to individual differences in the tendency to engage in facial mimicry. Studies of individuals with empathic disorders, such as ASD (Autistic Spectrum Disorder) or DBD (Disruptive Behaviour Disorder) also showed less mimicry when confronted with angry and sad faces. Mimicry has also been related to attachment behaviours, showing that insecurely attached children mimic positive emotions less often than securely attached children. The relation between empathy and mimicry is most likely bidirectional, however, because a variety of studies have also shown that mimicry affects liking, and bonding with the other person. Stel (Chapter 2) and Hess and colleagues (Chapter 5) describe studies showing that a priori attitudes influence mimicry, such that a positive attitude enhances mimicry, whereas a negative attitude decreases mimicry, but also that mimicking in turns increases positive attitudes, and thus may reinforce an empathic stance.

The relation between mimicry and empathy is therefore a close one. Most authors define empathy as a broad state of mind aimed at feeling or sharing with others, whereas mimicry is a more specific tendency to copy others' behaviour. We think it is important to differentiate the two concepts, while at the same time to acknowledge that they mutually influence each other.

\section{Functions of emotional mimicry}

Two functions of mimicry have traditionally been proposed and examined. The first is improving the understanding of others, which is discussed elaborately by Stel in Chapter 2. Several theories have provided explanations of this function, such as the Facial Feedback Hypothesis, the Perception-Behaviour link, and Embodiment theories, all suggesting that mimicry helps us to identify others' feelings. This seems particularly to be the case when others' feelings are not straightforward, and therefore difficult to unravel. In such cases differences between people who do or do not mimic are found. This is also evident from Chapter 3 by Niedenthal and colleagues who focus on smile mimicry. Their research on the role of smile mimicry has shown that participants whose mimicry is blocked are worse in detecting the distinction between true and false smiles. However, in general the results with regard to this function seem to be inconclusive. Whereas some studies found support for the facilitation or improvement of accuracy in recognizing others' emotions, other studies found no effects. Explanations for this inconsistency are discussed by Stel in Chapter 2.

A second function that has received much empirical support is the affiliation function: mimicry is assumed to lead to stronger and more 
positive social bonds. This is not only the case for smiling but also for negative emotions. Hess and colleagues review the evidence for social context effects of facial mimicry in Chapter 5 and show that mimicry is modulated by the social relational context in which it occurs. Individuals are more likely to mimic friends or people they like and less likely to mimic people they do not like or who are dissimilar to them. In addition, when individuals have the goal to cooperate, they are more likely to mimic others than when they are in competition. Indeed, Winkielman and colleagues (Chapter 8) further extend these social context effects of mimicry using human-like androids. This research shows that androids are mimicked but only in a cooperative and not in a competitive context. This research also emphasizes the human-bonding function, showing that the more human-like the android appears to be and the closer its presence, the more it was mimicked.

\section{The nature of mimicry: underlying processes}

Different theoretical approaches to explaining mimicry are reviewed in the various chapters. Most theories, such as the Matched Motor hypothesis, the Perception Action Model, and other variants of embodiment theory and simulation theory, assume that the observation of a facial expression elicits some form of internal simulation that is reflected in the activation of motor areas in the brain similar to the ones that are activated when one experiences the emotion oneself. Differences between these theoretical accounts relate to how the emotional signal is interpreted, what type of information needs to be processed, and at what level, in order for mimicry to occur. In Chapter 6 Hawk and Fischer discuss these different theoretical accounts extensively in order to explain the phenomenon of cross-channel mimicry, that is, the facial mimicry in response to auditory signals.

In contrast to views that assume an internal simulation of the perceived emotion, emphasizing self-other similarities, Schilbach (Chapter 4) proposes a second view that does not emphasize similarity with and matching of others' minds but instead an interactionist or enactive view of mimicry. In this account, knowledge of others' minds is required to support interpersonal coordination. He argues, in line with others in the volume, that mimicry based on the mirror neuron network (MNN) reflects the tight association between perception and premotor and motor areas in the brain, whereas mimicry based on the mentalizing network (MENT) is activated when socially relevant stimuli are processed more explicitly. Such a view is in line with our proposed Emotional Mimicry in Social Context model (see also Chapter 10, this book), which argues that emotional mimicry is not based on mere 
objective features of the face or the body but rather on the meaning and interpretation of these movements in a particular context.

\section{In sum}

These are just some of the many issues that are discussed in this volume. We hope that the volume will be useful and stimulate new research on the intriguing questions of how, why, and when we mimic others' emotions. Finally, we would like to thank all the authors for their enthusiasm to contribute to this volume, and we hope that they will enjoy the outcomes of this joint endeavour as much as we do.

\section{References}

Donald, M. (1991). Origins of the modern mind. Cambridge, MA: Harvard University Press.

Hatfield, E. C., Cacioppo, J. T., \& Rapson, R. L. (1994). Primitive Emotional contagion. Cambridge: Cambridge University Press.

Hess, U., \& Fischer, A. (2013). Emotional mimicry as social regulation. Personality and Social Psychology Review, 17, 142-157. 